\title{
A Single-mode Echelle Spectrograph: Eliminating Modal Variation, Enabling Higher Precision Doppler Study
}

\author{
Askari Ghasempour $^{a}$, John Kelly ${ }^{b}$, Matthew W. Muterspaugh ${ }^{a, b}$, Michael H. Williamson ${ }^{a}$ \\ ${ }^{a}$ Tennessee State University, Center of Excellence in Information Systems, 3500 John \\ A. Merritt Blvd., Box No. 9501, Nashville, TN 37209-1561, USA; \\ ${ }^{b}$ Department of Mathematical Sciences, College of Engineering, Tennessee State University, \\ Boswell Science Hall, Nashville, TN 37209
}

\begin{abstract}
An innovative compact - yet high resolution - cross-dispersed echelle spectrograph has been designed, built, and deployed at TSU's 2-meter robotic telescope for initial tests and commissioning. This design is based on a single mode fiber (SMF) and it eliminates mode noise in fiber-fed spectrographs which is important for $\mathrm{m} / \mathrm{s}$ precision exoplanet Doppler searches. The use of SMFs removes modal variation, makes the design compact and the camera focus slow and stable at the price of lower throughput. This can be improved by using adaptive optics or by placing it in space; the compact design is well suited for such deployment.
\end{abstract}

Keywords: instrumentation: spectrographs, instrumentation: adaptive optics, instrumentation: fiber optics

\section{INTRODUCTION}

Precision Doppler Velocimetry has many applications in astronomy, with the most recent being the emergence of the field of exoplanet exploration. In the 1970's through 1980's, measurement precisions of 10's of m/s on bright stars were demonstrated using first telluric features,${ }^{1}$ then HF gas cells, ${ }^{2}$ for wavelength and instrument response function calibration. These efforts gave the first hints of planetary companions to nearby stars. ${ }^{3}$ In the 1990's several teams migrated to $I_{2}$ gas absorption cells for calibration, improving the observed precision to $3 \mathrm{~m} \mathrm{~s}^{-1}{ }^{4}$ This improvement brought about the initial confirmed exoplanet discoveries around nearby Sunlike stars, and marked the beginning of exoplanet systems being discovered in large numbers. Today, measurement precisions of $\sim 1 \mathrm{~m} \mathrm{~s}^{-1}$ represent the state-of-the-art using instruments such as HIRES at Keck Observatory ${ }^{5,6}$ and HARPS,${ }^{7,8}$ which use $\mathrm{I}_{2}$ gas absorption cells and/or ThAr emission lamps for calibration. Each factor of $\frac{1}{2}$ order of magnitude increase in precision has enabled new science regimes, as is so often the case in astronomy.

The limiting factor with HIRES and HARPS is believed to be instrumental in nature, due to the nonrepeatability of the entrance image's illumination of the spectrographs' slits. At HIRES, a direct-fed spectrograph, this is due to slit wander from seeing and telescope guiding. For HARPS, fed by a multimode fiber, a similar effect is caused by starlight wandering on the fiber's entrance, and mechanical/thermal agitation of the fiber itself. Considering the fiber as an optical waveguide, variable coupling to the fiber leads to propagation modes being illuminated with variable amounts of power. This is combined with the natural tendancy for modes to transfer power from one to the other in a manner sensitively dependent on the details of the fiber bends and stresses, which cause the power distribution among the modes at the fiber output to have variable patterns. While azimuthal modes in fibers scramble well and generally are evenly illuminated, obtaining similar scrambling efficiency for radial modes is more challenging. ${ }^{9}$ A number of solutions have been proposed, including active fiber agitation and double-scrambling lenses, ${ }^{10-12}$ and are being tested, but these at-best reduce the effect rather than eliminating it altogether.

Should one be able to overcome these instrumental limitations, the ultimate achievable Doppler precision attainable is still a matter of some debate. At some point, the stars themselves contribute astrophysical noise in

Further author information: (Send correspondence to M.W.M.)

M.W.M.: E-mail: matthew1@coe.tsuniv.edu, Telephone: 16179562753

A.G.: E-mail: askari@coe.tsuniv.edu

Modern Technologies in Space- and Ground-based Telescopes and Instrumentation II,

edited by Ramón Navarro, Colin R. Cunningham, Eric Prieto, Proc. of SPIE Vol. 8450, 845045

(C) 2012 SPIE · CCC code: 0277-786X/12/\$18 · doi: 10.1117/12.925031

Proc. of SPIE Vol. $8450845045-1$ 
the form of "jitter" - surface motions including quasi-coherent pulsations that may dominate any measurement effort. There is some speculation that a small fraction of stars will exhibit so little jitter (or that full-cycles of modes can be averaged) that a sufficiently stable spectrograph could produce Doppler measurements an order of magnitude better than current capabilities, enabling a search for true Earth analogs (Doppler signal $\sim 10 \mathrm{~cm} \mathrm{~s}^{-1}$ ) to be performed on those stars. ${ }^{13}$ If any such stars do exist, they would almost certainly be a small fraction of all targets of interest, but would enable an initial finding program for much less expense than the alternatively proposed major space-based observatories such as TPF or an astrometric mission.

Thus, the question remains, how stable are the most stable of stars? Do the properties of the stars themselves even permit a search for true Earth analogs using the Doppler technique? To answer this, an instrument with instrinsic stability is required that overcomes the systematic limitations present in HIRES and HARPS, even if one must trade other considerations to achieve that goal. For example, one can initially concentrate on just the brightest stars to evaluate their characteristics, trading sensitivity design considerations for point-spread-function stability. Fortunately, there are a few bright stars that current instruments indicate have intrinsic noise levels at least as small as the instruments' noise floors. These include $\tau$ Ceti $(V=3.5)$ and $\sigma$ Draconis $(V=4.68)$.

One option for trading throughput for image stability is to use single mode fibers. Because only the fundamental mode of the optical waveguide is allowed to propagate, the exit image necessarily is shaped as that mode, and unchanging. This uniformity and repeatability makes a single mode fiber a perfect fiber scrambler, eliminating this systematic. However, the small size of the fiber causes it to accept very little starlight. To our knowledge, no one has built such an instrument; the basic idea was proposed by Connes et al. but never developed. ${ }^{9}$ In this paper we present the design and first results from a cross-dispersed, high resolution echelle spectrograph fed by a single mode fiber. In our initial effort, we have not introduced additional instrument stabilization measures such as vacuum, pressure control, or temperature control systems, a choice which may reintroduce other systematic errors; these will be added as needed upon iteration with the first results of the instrument's stability.

\section{DESIGN CONSIDERATIONS}

\subsection{Velocity Precision Factors}

In designing a Doppler instrument, maximization of velocity precision is desired. The calculation of the photonnoise limited velocity precision starts with Bouchy et al.'s ${ }^{14}$ equation 16

$$
\delta V=\frac{c}{Q \sqrt{F S e}}
$$

where $Q$ is a quality factor of the spectrum (a quantitative measure of the Doppler information content due to the shape of the spectrum), $F$ is the flux from the star in photons $\mathrm{m}^{-2} \mathrm{~s}^{-1}, S$ is the telescope's collecting area, and $e$ is the telescope $\times$ instrument $\times$ sky throughput. Following their discussion, $Q$ depends on the star's spectral type, the star's projected rotational velocity $v \sin i$, and the spectrograph resolution $R$. The zero-rotational-velocity, infinite resolution $Q$ factor for a given spectral type, called $Q_{\circ}$, is modified to get $Q$ as follows: $Q$ scales inversely with $\sqrt{v \sin i}$ (note there is an error in the original source paper which omits the radical) and proportionally to $R$ (up to a resolution of about 100,000). The flux $F$ is further dependent on instrument bandwidth; $F=N B$, where $N$ is the (instrument independent) flux per bandwidth in photons $\mathrm{m}^{-2} \mathrm{~s}^{-1} \AA^{-1}$ and $B$ is the (instrument dependent) bandwidth in $\AA$. Thus, one derives the following relationship for predicting photon noise limited velocity performance

$$
\begin{aligned}
\sigma & \propto\left(\frac{1}{\mathrm{~N}^{1 / 2}} \frac{1}{R} \frac{1}{B^{1 / 2}} \frac{1}{Q_{\circ}}(v \sin i)^{1 / 2}\right) \\
& \approx 0.21 \mathrm{~m} \mathrm{~s}^{-1}\left(\frac{10^{5}}{R}\left(\frac{1400 \AA}{B}\right)^{1 / 2} \frac{30000}{Q_{\circ}}\left(\frac{v \sin i}{1 \mathrm{~km} \mathrm{~s}^{-1}}\right)^{1 / 2}\left(\frac{0.06 \mathrm{~m}}{D_{\text {tel }}}\right)^{1 / 2}\left(\frac{1}{e}\right)^{1 / 2}\left(\frac{60 \mathrm{~s}}{t}\right)^{1 / 2} 10^{-V / 5}\right)
\end{aligned}
$$

where $Q_{\circ} \sim 30000$ for late $\mathrm{G} /$ early $\mathrm{K}$ stars, and $D_{\text {tel }}$ is the effective telescope diameter (the choice of $6 \mathrm{~cm}$ is justified for any larger uncorrected telescope coupled to a single mode fiber ${ }^{15}$ ). The bandwidth $B$ depends on the calibrant used (an iodine gas absorption cell; $\sim 1400 \AA$ ). Thus, we need to maximize the resolution. 


\subsection{Bandpass and Calibration}

Because velocity precision increases as more of the spectrum is collected and depends on the quality of the spectral features within the range of wavelengths selected, choosing the operating bandpass is a crucial first step. ThAr emission lamps and gaseous $\mathrm{I}_{2}$ absorption cells are commonly used, with laser combs being promising future technology. ${ }^{16}$ All of these offer sufficient spectral information to be used for precision velocity calibration in the applicable wavebands. An advantage to ThAr and laser combs is that they can be used over a very broad range of wavelengths, as compared to $\mathrm{I}_{2}$, which is limited to $\sim 4800-6200 \AA$. However, $\mathrm{I}_{2}$ has the advantage of being an absorptive calibrant, imprinting its spectrum into the starlight itself, and the starlight carries this calibration with it throughout the instrument. In contrast emission sources - even when recorded on the same pixels as the starlight - can sample different parts of the optics than the starlight itself in their paths to the detector, leading to calibration errors. As a prototyping effort, we have chosen $\mathrm{I}_{2}$ as the calibrant to reduce the possibility of introducing such errors and simplifying the number of possible problems that will need to be explored. This sets the operating bandpass at $\sim 4800-6200 \AA$.

\subsection{Spectral Resolution Considerations}

Three factors limit spot size and thus resolution. Each needs to be minimized. First, maximizing the dispersion of the grating element is needed to spread the light most completely and measure the sharpest features. Second, diffraction (as in the imaging limit of a given optic diameter, not that due to a diffraction grating) can be an important factor; normally this is ignored in echelle spectrographs because they have relatively large slits and low $\mathrm{f} / \#$ cameras, though that is not the present case. Finally, optical aberrations due to imperfect elements need to be reduced by optical design and use of multiple correcting elements; as will be seen, the properties of the compact spectrograph already minimize these.

Dispersion: Given a fixed number of pixels in the high resolution dimension of the detector, and assuming one matches the image spot size to the pixel size, resolution is maximized by having as small a bandwidth as possible in each order. To avoid gaps in the spectrum, one must make the order-to-order separation in wavelength as small as possible. The order spacing from a diffraction grating is

$$
\delta \lambda=\frac{\lambda_{\circ}^{2} g}{2 \sin \theta_{b}}
$$

where $\lambda_{\circ}$ is the central wavelength, $g$ is the number of lines per unit length, and $\theta_{b}$ is the blaze angle of the grating. An optimal grating is one that minimizes $g / \sin \theta_{b}$. Inspection of the standard Richardson Gratings at Newport Optics reveal that two selections minimize this the best: 53 - $^{*} 412 \mathrm{E}\left(g=23.2 \mathrm{l} / \mathrm{mm}, \theta_{b}=63^{\circ}\right)$ and 53 $*_{-} 419 \mathrm{E}\left(g=24.35 \mathrm{l} / \mathrm{mm}, \theta_{b}=70^{\circ}\right)$. For ease of use, the one with lower blaze angle is chosen. Newport's smallest standard grating size is $50 \times 100 \times 16 \mathrm{~mm}$, which will turn out to be sufficient for this prototype instrument.

With the grating selected, the set of orders is identified: $m=124$ through $m=160$ span $4800-6200 \AA$. These 37 orders set a lower limit on the size of one's detector in the cross-dispersing direction: if one desires $\sim 6-12$ pixels between adjacent orders, a CCD with 256 or 512 pixels in that dimension is required.

There is a trade-off between resolution and cutting off the edges of the order where the grating efficiency starts tapering off. A Gaussian serves as an approximate model of the grating efficiency fall-off:

$$
f(\lambda)=A e^{-\left(\lambda-\lambda_{\circ}\right)^{2} /(0.55 \delta \lambda)^{2}}
$$

where $A$ is the peak efficiency (around 70\%), $\delta \lambda$ is the order-to-order spacing, and 0.55 is an estimate based on published efficiency curves for the selected grating. For the best velocity precision, one should maximize $\sqrt{F R^{3} B}$, where $F$ is flux. The flux received times effective bandwidth $(N B)$ is proportional to $\Lambda$ (the single order spectral range, limited by the size of the detector in the high-resolution dimension) and the efficiency curve integrated over the spectral range. The resolution $R$ is proportional to $1 / \Lambda$. The best velocity precision is possible when one maximizes:

$$
\int_{\lambda_{\circ}-\Lambda / 2}^{\lambda_{0}+\Lambda / 2} \sqrt{f(\lambda) / \Lambda} d \lambda
$$


which increases as $\Lambda \rightarrow 0$; resolution always wins. However, this is only accurate to a point; stellar rotation broadens the lines of even the slowest rotators to $2-3 \mathrm{~km} \mathrm{~s}^{-1}$, so the approximation that $R$ scales as $1 / \Lambda$ breaks down around $R \sim 10^{5}$. Choosing $\Lambda=40 \AA$ (the approximate order-to-order spacing) collects most of the light (81\%) while preserving $R \sim 140,000$ with a 2048 pixel detector at 2-pixel sampling.

The target spectral resolution determines the diameter $B$ of the collimated beam in the spectrograph by the echelle throughput equation ${ }^{17}$

$$
R=\frac{2 B \tan \theta_{b}}{D S}
$$

where $D$ is the telescope diameter and $S$ the acceptance angle of the slit. For light from a single mode fiber, $D S$ should be replaced by $z a / f \#$, where $a$ is the mode field diameter of the fiber, $z \sim 1.5$ is a factor converting $a$ to the full image size (a subtle difference), and $f \#$ is the focal ratio of light exiting the fiber, related to the numerical aperture as $\left(\sqrt{1 / \mathrm{NA}^{2}-1}\right) / 2$. NuFern produces standard single mode fibers with cutoff wavelengths around $4300 \AA$; these are the closest to the target bandpass. The mode field diameter is $a=3.4 \mu \mathrm{m}$ and $\mathrm{NA}=0.12$. The beam diameter is

$$
B=\frac{R z a}{\tan \theta_{b} \sqrt{1 / \mathrm{NA}^{2}-1}} \sim 45 \mathrm{~mm} .
$$

This sets the input focal length of the spectrograph:

$$
f_{\text {in }}=B\left(\sqrt{1 / \mathrm{NA}^{2}-1}\right) / 2 \sim 186 \mathrm{~mm} .
$$

Diffraction: The size of the spectrograph optics diffract the image spot size to

$$
\Delta x=1.22 \frac{\lambda}{B} f_{\text {out }}
$$

where $\lambda$ is the wavelength of the light, $B$ is the diameter of the collimated beam in the spectrograph, and $f_{\text {out }}$ is the focal length of the camera in the spectrograph. Normally, echelle spectrographs have large beam diameters (to illuminate many lines on the grating for high spectral dispersion), and fast cameras (small $f_{\text {out }}$ ) because the input image size is much larger than a pixel on typical CCD cameras, since the image is enlarged by seeing. These are not the case here.

The beam size is related to the collimator's focal length $f_{\text {in }}$ and the $f / \#$ of the light emerging from the fiber.

$$
B=\frac{f_{\text {in }}}{f_{\#, \text { in }}}=\frac{2 f_{\text {in }}}{\sqrt{1 / \mathrm{NA}^{2}-1}}
$$

Thus,

$$
\Delta x=0.61 \lambda \sqrt{1 / \mathrm{NA}^{2}-1}\left(f_{\text {out }} / f_{\text {in }}\right)=2.8\left(f_{\text {out }} / f_{\text {in }}\right) \mu \mathrm{m} \text { at } 5500 \AA
$$

The output focal length is set by matching the overall image size to equal 2 pixels. We had a spare Andor iDus 420 spectroscopy camera at TSU which we repurposed for this project. Its detector has $1024 \times 256$ pixels each $26 \mu \mathrm{m}$ on a side. Andor also produces the Newton 940N, which has twice as many pixels in each dimension with nearly half the size $(13.5 \mu \mathrm{m}$ on a side). To save cost, the spectrograph was first built using the spare iDus, but designed to be optimized for a potential future upgrade to the Newton; the loss in going from 2-pixel to 1-pixel sampling simply reduces the spectral resolution. The total spot size is determined by the size of the magnified fiber tip and the extra diffraction from the finite sized beam

$$
\Delta x_{\mathrm{tot}}=\left(z a+0.61 \lambda \sqrt{1 / \mathrm{NA}^{2}-1}\right) \frac{f_{\text {out }}}{f_{\text {in }}}
$$

Setting this to $27 \mu \mathrm{m}$ determines a target output focal length of $\sim 638 \mathrm{~mm}$. 
A camera with good noise properties but smaller pixels would further reduce the size of the instrument (which is dominated by the long camera focal length; the dispersive optics themselves fit in a package under 12" on a side), but an initial search has not identified any candidates. Continued efforts in this direction are of potential interest for non-astronomy applications. This can result in an entire instrument package enclosed in a size of order $1 \mathrm{ft}^{3}$.

Unlike most spectrographs, this design magnifies the entrance image onto the detector, rather than demagnifying. This greatly simplifies the optical design. The fastest optics are on input, but the field of view is small (the size of the fiber optic), easing aberration requirements there. An off-axis parabola is an excellent choice. The large field of view is on output, where there are very slow optics, so even a simple doublet lens is sufficient to reduce aberrations. The 6-7 (or more) element, large field, ultra-fast cameras found in most echelle spectrographs are not needed in this design, restoring some throughput and greatly improving simplicity and cost.

\subsection{Cross-Dispersion}

With the focal length of the output set, one can choose a cross-dispersing element. This needs to spread light from $4800-6200 \AA$ across a distance of $\sim 512$ pixels each $13.5 \mu \mathrm{m}$ high, which corresponds to $280 \AA / \mathrm{mm}$. A $60^{\circ}$ BK7 prism is sufficient, and has the added benefit that the orders have roughly uniform spacing.

\subsection{Final Considerations}

For simplicity, reliability, and in the interest of keeping costs low, off-the-shelf components were selected when possible. As a result, the final configuration had: $f_{\text {in }}=134 \mathrm{~mm}$ (based on Edmunds Optics NT90-976 - an off-the-shelf off-axis parabola), a slightly smaller collimated beam size $B=32.4 \mathrm{~mm}$-which better fits the grating size, $f_{\text {out }}=500 \mathrm{~mm}$ (this allows slightly more of each order to fall on the detector, and with the current camera, no resolution loss is suffered since it is already pixel size limited), spot size $\Delta x_{\text {tot }}=29.4 \mu \mathrm{m}$, an opticslimited resolution $R \sim 70,000$, and pixel limited resolution (with the current CCD) $R \sim 40,000$. We own a spare $f \sim 165 \mathrm{~mm}$ off-axis parabola from the VISION project, ${ }^{18}$ which may be used in the future to enhance this design. Also, a $f_{\text {out }} \sim 750 \mathrm{~mm}$ lens may be explored to better match pixel sampling, especially with the alternative collimator.

A Thorlabs FC532-90B-FC 90/10 fiber coupler was connected to the single mode fiber to redirect some of the starlight to a single pixel detector as an exposure monitor. The optics path is then as follows: light from the fiber is collimated by the $f=134 \mathrm{~mm}$ off-axis parabola. At the proper pupil of the parabola (134 $\mathrm{mm}$ further) is the center of the echelle grating. The echelle is used "in-plane" $(\gamma=0)$ with just enough of an angle to have the return beam miss the off-axis parabola. This increased the beam size somewhat, so the previous numbers for spot size and resolution are on the pessimistic side. The dispersed but still collimated light is reflected back on itself by a flat mirror, but with an angle pointing it out of the original plane, and it enters the cross-dispersing prism, which once again makes the light parallel to the original plane, though displaced vertically. This solves the problem of the cross-disperser creating an out-of-plane angle. The cross-disperser is not at a proper pupil, however. The camera lens is an off-the-shelf doublet and is placed at a point that is optically a distance $f_{\text {in }}+f_{\text {out }}$ from the collimator, so the echelle is at a pupil of both. From there the light goes to the CCD detector.

\subsection{Other Fortunate Consequences of Restricting to Single-Mode Operation}

The small size of the single mode fiber leads to several favorable design features. First, the overall size of the spectrograph is small. This leads to an intrinsic stability and allows it to be easily transportable, which is favorable to some non-astronomical applications where a mobile instrument is desired. Already mentioned is that the spectrograph is magnifying rather than demagnifying, placing the fast optics (with the largest off-axis aberrations) where the field of view is small, and slow optics at the output where the field is large. Simple off-the-shelf single or double element optics provide sufficient image qualities. In addition, the long focal length of the output optic simplifies alignment and increases focus tolerances. It is easy to reconfigure the instrument for different telescopes. This is crucial for the envisioned future uses of the instrument at telescopes equipped with adaptive optics, which will increase the efficiency that starlight couples to the single mode fiber. This application of adaptive optics technology has considerable benefits - all that is desired is to increase the amount of light entering the fiber; image errors such as speckles do not hurt the science program. Indeed, a major science 


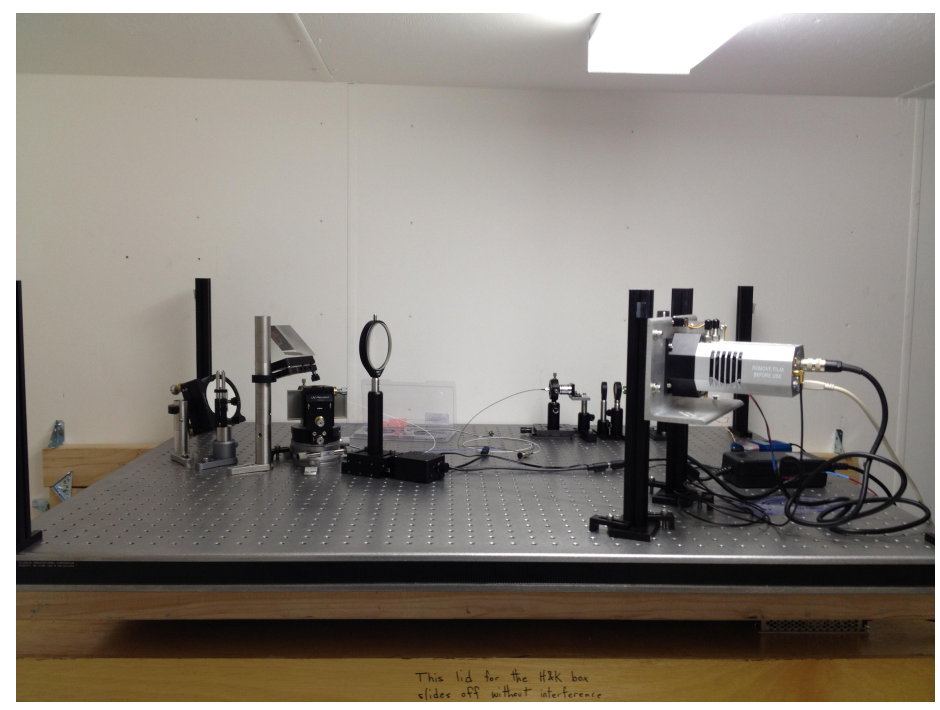

Figure 1. The echelle spectrograph installed at Fairborn Observatory with fiber feed from the AST.

opportunity for adaptive optics units is in the field of spectroscopy rather than high resolution imaging because image artifacts which cause calibration problems for imaging can be ignored. Also, the adaptive optics restoration of image sharpness lessens the amount of sky to which the spectrograph is sensitive, reducing background light. This is potentially of interest for faint object spectroscopy.

\section{PARTS AND COST}

All parts used to build the instrument were standard, and most were off-the-shelf items produced in large quantities. The only custom components were the fiber patch cord (a standard item, only the length and a stainless steel jacket had to be specified), the grating (also a standard item based on the Richardson Grating selections, just not produced in large quantities), and the cross-dispersing prism (only unusual due to its 3" size). The total cost, excluding camera and optical breadboard (which were already owned), was under $\$ 10,000$.

\section{DEPLOYMENT AT TSU AST}

After construction and testing in the lab, the spectrograph was delivered to Fairborn Observatory for use with Tennessee State University's 2-meter robotic Automatic Spectroscopic Telescope (AST; see Figure $1^{19}$ ). The AST is quite oversized for the instrument, since any telescope larger than the atmospheric coherence length $\left(r_{\circ} \sim 6 \mathrm{~cm}\right)$ without adaptive optics will couple only as much light into a single mode fiber as a telescope of that smaller size. ${ }^{15}$ This platform was instead chosen for its accessibility and the ease of robotic operations during prototype testing and commissioning. Switching between the many fiber-fed instruments at the AST requires a matter of seconds, demonstrating the AST's flexibility for observing cadence and instrument prototyping. ${ }^{20}$ First light was acheived in early 2012.

Figure 2 shows a first-light spectra of Pollux ( $V=1.15,1$ hour exposure). The spectrum has been roughly normalized and orders are offset vertically by order number; orders 115-158 are shown, covering a wavelength range of $\sim 4850-6700 \AA$ simultaneously. Sodium $\mathrm{D}$ features are readily identified in order 130 , and $\mathrm{H}_{\alpha}$ in order 117. The orders overlap considerably; given the current detector pixel size and order spacing, a slightly longer output focal length of $\sim 750 \mathrm{~mm}$ would have been preferable and may be implemented in the near future. The SNR at the middle order is estimated to be $\sim 115$. This is an order of magnitude below the predicted amount for such a bright star, and is probably due to imperfect optics in the AST primary-secondary mirror system; a new secondary mirror is expected to be deployed in summer 2012. Lab-based estimates of the fiber-to-CCD throughput show the spectrograph itself is relatively high throughput $(>50 \%)$. 


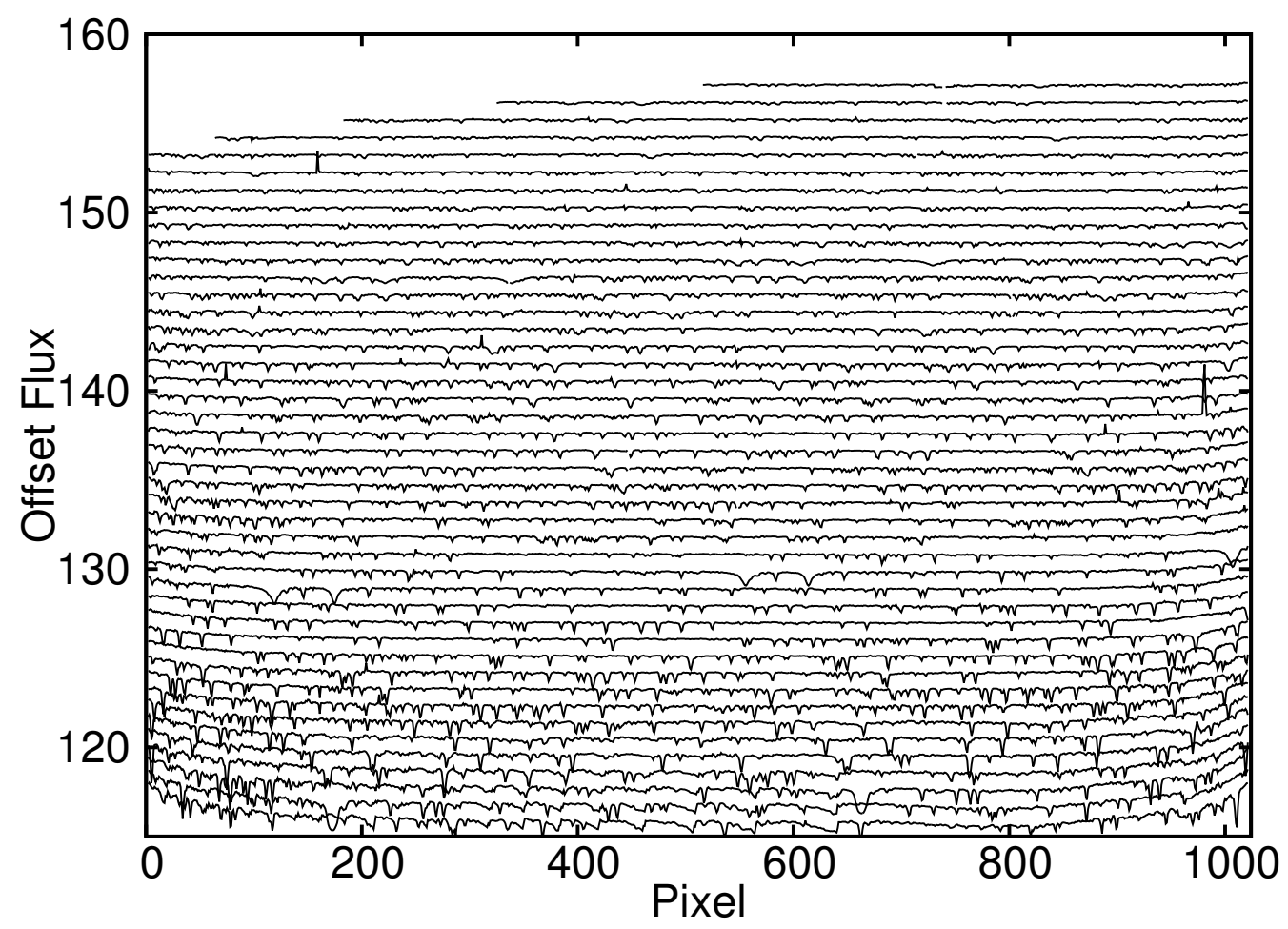

Figure 2. First light spectrum of Pollux.

\section{FUTURE DIRECTIONS}

An iodine calibration cell is being added to the telescope concurrent with this conference. This will enable the instrument's stability to be explored, and is available for use with the AST's other instruments. Once the instrument performance is demonstrated on bright stars, increased throughput for the sensitivity required of a general survey can be obtained using an adaptive optics unit to restore the seeing-degraded image quality of the telescope. This will likely require transfering the instrument to an alternative telescope.

\section{ACKNOWLEDGMENTS}

This work was supported by a EPSCoR subcontract from Vanderbilt University and the State of Tennessee through the Centers of Excellence program. We thank Cullen Blake, Phillip Muirhead, John Johnson, and Maciej Konacki for helpful discussions about this project.

\section{REFERENCES}

[1] Griffin, R. and Griffin, R., "Accurate wavelengths of stellar and telluric absorption lines near lambda 7000 Angstroms," MNRAS 162, 255 (1973).

[2] Campbell, B. and Walker, G. A. H., "Precision radial velocities with an absorption cell," PASP 91, 540-545 (Aug. 1979).

[3] Campbell, B., Walker, G. A. H., and Yang, S., "A search for substellar companions to solar-type stars," ApJ 331, 902-921 (Aug. 1988).

[4] Butler, R. P., Marcy, G. W., Williams, E., McCarthy, C., Dosanjh, P., and Vogt, S. S., "Attaining Doppler Precision of 3 M s-1," PASP 108, 500 (June 1996). 
[5] Vogt, S. S., Allen, S. L., Bigelow, B. C., Bresee, L., Brown, B., Cantrall, T., Conrad, A., Couture, M., Delaney, C., Epps, H. W., Hilyard, D., Hilyard, D. F., Horn, E., Jern, N., Kanto, D., Keane, M. J., Kibrick, R. I., Lewis, J. W., Osborne, J., Pardeilhan, G. H., Pfister, T., Ricketts, T., Robinson, L. B., Stover, R. J., Tucker, D., Ward, J., and Wei, M. Z., "HIRES: the high-resolution echelle spectrometer on the Keck 10-m Telescope," in [Society of Photo-Optical Instrumentation Engineers (SPIE) Conference Series], Crawford, D. L. and Craine, E. R., eds., Society of Photo-Optical Instrumentation Engineers (SPIE) Conference Series 2198, 362 (June 1994).

[6] Howard, A. W., Johnson, J. A., Marcy, G. W., Fischer, D. A., Wright, J. T., Henry, G. W., Giguere, M. J., Isaacson, H., Valenti, J. A., Anderson, J., and Piskunov, N. E., "The NASA-UC Eta-Earth Program. I. A Super-Earth Orbiting HD 7924," ApJ 696, 75-83 (May 2009).

[7] Pepe, F., Mayor, M., Delabre, B., Kohler, D., Lacroix, D., Queloz, D., Udry, S., Benz, W., Bertaux, J.-L., and Sivan, J.-P., "HARPS: a new high-resolution spectrograph for the search of extrasolar planets," in [Society of Photo-Optical Instrumentation Engineers (SPIE) Conference Series], Iye, M. and Moorwood, A. F., eds., Society of Photo-Optical Instrumentation Engineers (SPIE) Conference Series 4008, 582-592 (Aug. 2000).

[8] Lovis, C., Pepe, F., Bouchy, F., Lo Curto, G., Mayor, M., Pasquini, L., Queloz, D., Rupprecht, G., Udry, S., and Zucker, S., "The exoplanet hunter HARPS: unequalled accuracy and perspectives toward $1 \mathrm{~cm} \mathrm{~s}^{-1}$ precision," in [Society of Photo-Optical Instrumentation Engineers (SPIE) Conference Series], Society of Photo-Optical Instrumentation Engineers (SPIE) Conference Series 6269 (July 2006).

[9] Connes, P., Martic, M., and Schmitt, J., "Demonstration of Photon-Noise Limit in Stellar Radial Velocities," ApSS 241, 61-76 (Mar. 1996).

[10] Connes, P., "Absolute astronomical accelerometry," ApSS 110, 211-255 (Mar. 1985).

[11] Hunter, T. R. and Ramsey, L. W., "Scrambling properties of optical fibers and the performance of a double scrambler," PASP 104, 1244-1251 (Dec. 1992).

[12] Brown, T. M., Noyes, R. W., Nisenson, P., Korzennik, S. G., and Horner, S., "The AFOE: A spectrograph for precise Doppler studies," PASP 106, 1285-1297 (Dec. 1994).

[13] Torres, G., Gelino, D. M., von Braun, K., and Forum 2008 Radial Velocity Committee, E., "Exoplanet Community Report on Radial Velocities," in [American Astronomical Society Meeting Abstracts \#213], Bulletin of the American Astronomical Society 41, 310.06 (Jan. 2009).

[14] Bouchy, F., Pepe, F., and Queloz, D., "Fundamental photon noise limit to radial velocity measurements," A 6 A 374, 733-739 (Aug. 2001).

[15] Shaklan, S. and Roddier, F., "Coupling starlight into single-mode fiber optics," AO 27, 2334-2338 (June 1988).

[16] Wilken, T., Curto, G. L., Probst, R. A., Steinmetz, T., Manescau, A., Pasquini, L., González Hernández, J. I., Rebolo, R., Hänsch, T. W., Udem, T., and Holzwarth, R., "A spectrograph for exoplanet observations calibrated at the centimetre-per-second level," Nature 485, 611-614 (May 2012).

[17] Vogt, S. S., "The Lick Observatory Hamilton Echelle Spectrometer," PASP 99, 1214-1228 (Nov. 1987).

[18] Ghasempour, A., Muterspaugh, M. W., Hutter, D. J., Monnier, J. D., Benson, J. A., Armstrong, J. T., Williamson, M. H., Fall, S., Harrison, C., and Sergeyous, C., "Building the next generation science camera for the Navy optical interferometer," in [Proc. SPIE], Presented at the Society of Photo-Optical Instrumentation Engineers (SPIE) Conference (2012).

[19] Eaton, J. A. and Williamson, M. H., "The Tennessee State University Automatic Spectroscopic Telescope: Data Processing and Velocity Variation of Cool Giants," PASP 119, 886-897 (Aug. 2007).

[20] Muterspaugh, M. W., Williamson, M. H., Fekel, F. C., and Harrison, C., "The Upgraded Tennessee State University 2m Automatic Spectroscopic Telescope," in [American Astronomical Society Meeting Abstracts], American Astronomical Society Meeting Abstracts 219, 422.04 (Jan. 2012). 